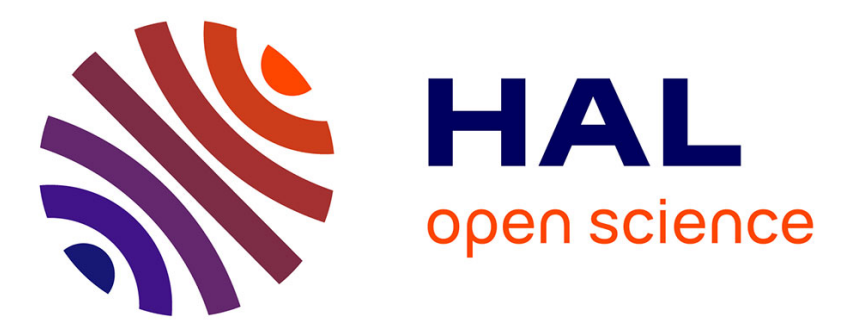

\title{
Residual Stresses in Metallic Multilayers
}

\author{
O. Thomas, P. Gergaud, S. Labat, L. Barrallier, A. Charaï, Claude Alfonso, \\ B. Gilles, A. Marty
}

\section{To cite this version:}

O. Thomas, P. Gergaud, S. Labat, L. Barrallier, A. Charaï, et al.. Residual Stresses in Metallic Multilayers. Journal de Physique IV Proceedings, 1996, 06 (C7), pp.C7-125-C7-134. 10.1051/jp4:1996714 . jpa-00254502

\section{HAL Id: jpa-00254502 https://hal.science/jpa-00254502}

Submitted on 1 Jan 1996

HAL is a multi-disciplinary open access archive for the deposit and dissemination of scientific research documents, whether they are published or not. The documents may come from teaching and research institutions in France or abroad, or from public or private research centers.
L'archive ouverte pluridisciplinaire HAL, est destinée au dépôt et à la diffusion de documents scientifiques de niveau recherche, publiés ou non, émanant des établissements d'enseignement et de recherche français ou étrangers, des laboratoires publics ou privés. 


\title{
Residual Stresses in Metallic Multilayers
}

\author{
O. Thomas, P. Gergaud, S. Labat, L. Barrallier*, A. Charaï**, C. Alfonso**, B. Gilles*** and \\ A. Marty***
}

\author{
MATOP, URA 1530 du CNRS, Faculté de Saint Jérôme, 13397 Marseille cedex 20, France \\ * MécaSurf, CER ENSAM, 13617 Aix-en-Provence cedex 1, France \\ ** EDIFIS, URA 443 du CNRS, Faculté de Saint Jérôme, 13397 Marseille cedex 20, France \\ *** CEA/DRFMC/SP2M, Centre d'Études Nucléaires, 38054 Grenoble cedex 9, France
}

\begin{abstract}
The study of residual stresses in metallic superlattices is of great importance. Properties of these materials are a consequence of their extreme strain states. With respect to single films, both the determination methods and the magnitude of the stresses may differ in multilayers. X-ray strain determination must be used with caution because of possible interference effects. The large amount of disorder tends to suppress the modulation on asymmetrical peaks. Stress deduced from wafer bending experiments may contain a non negligible interfacial contribution. The very large stresses $(\geq 1 \mathrm{Gpa})$ determined in the superlattices imply very high yield stresses, possibly related to size effects. Anomalous strain free lattice parameters are frequently reported. They may be related to the occurrence of segregation during the growth.
\end{abstract}

\section{INTRODUCTION}

Superlattices are of considerable industrial interest because of their specific properties $[1,2]$ (magnetic, electronic, tribological, mechanical, optical, ...). Multilayer stackings are very often in a very high state of intrinsic stress (some GPa) and for reliable integrated devices to be made it is capital to control and understand these intrinsic as well as extrinsic stresses. Beyond these issues of reliability, stress and the resulting strain can be used to tune the properties of thin film materials. For instance, strain coupled with the magnetoelastic effect, can be utilized to induce the preferred magnetization direction [3]. One of the reasons which have been put forward for the anomalous mechanical properties of multilayered materials were the extreme strain states in the constituents. Indeed, the mechanical properties of multilayers have attracted interest because of the so-called « supermodulus effect »[4]: a very large enhancement (100\%) of the elastic moduli has been observed in a number of systems ( $\mathrm{Au}-\mathrm{Ni}, \mathrm{Cu}-\mathrm{Pd}, \mathrm{Ag}-\mathrm{Pd}, . .$. ) for short modulation wavelengths, of the order of $2 \mathrm{~nm}$. There is presently a growing consensus to attribute these results to an experimental artifact $[5,6]$. Finally, one should not forget that in these systems the stored mechanical energy is a non negligible part of the total energy. In particular the stress level can affect the miscibility [7] or even the stability of a given structure [8].

Many good review papers have been written on residual stresses in thin films [9-11]. This communication will focus more specifically on metallic multilayers, with an emphasis on the difference in behaviour with what is observed in thin films. and on the specific experimental strategies to be applied for multilayers.

\section{EXPERIMENTAL METHODS}

The most common ways to determine residual stresses in thin coatings are the measurement of the substrate bending and the measurement of interplanar spacings by x-ray diffraction. The important point here is the applicability of the methods to metallic multilayers. 


\subsection{Substrate bending}

A biaxially stressed film adhering to a substrate causes the film-substrate composite to deform elastically in biaxial bending. This mechanical equilibrium can be decribed in two different ways: either by writing the momentum and force balance [12-14] or by minimizing the total mechanical energy [15,16]. An important assumption is the elastic behaviour of the substrate which forbids the use of metallic substrates. All theses derivations agree for the thin film case, i.e. when the film thickness is much smaller than the substrate thickness. The corresponding expression is the Stoney formula:

$$
\sigma \mathrm{t}_{\mathrm{f}}=Y_{s} \frac{t_{s}^{2}}{6} \frac{1}{R}
$$

where $\sigma$ is the biaxial stress, $t_{f}$ the film thickness, $t_{s}$ the substrate thickness and $R$ the radius of curvature. $Y_{s}$ is the biaxial modulus of the substrate which depends on both the Young modulus E and the Poisson ratio $v$, both of the substrate: $Y_{s}=E /(1-v)$. For (100) silicon $Y_{s}=180 \mathrm{GPa}$ [17].

This expression shows that the film-substrate composite curvature is directly proportional to the force per unit length applied by the film on the substrate. From the knowledge of the film thickness one can then deduce the biaxial stress in the film. Because of the thin film approximation, i.e. because the flexural modulus of the composite is completely dominated by the substrate, the curvature does not depend on the elastic properties of the film. In the thin film limit the curvature method is thus a direct film stress measurement through the measurement of the substrate elastic strain. The bending strain in the film, of the order of $t_{s} / R$, is generally negligible compared to the residual strains.

One can distinguish two different ways of measuring the curvature, either by measuring the curvature of the substrate lattice planes or by measuring the surface curvature. The first method $[18,19]$ uses transmission $\mathrm{x}$-ray diffraction from the substrate lattice planes perpendicular to the surface. Its main disadvantage is that it is limited to single crystal substrates; it is rather slow which makes it unsuitable for in-situ measurements. It has the advantage to be relatively insensitive to the initial substrate topography because it measures directly the curvature of the lattice planes. The second class of methods relies generally on optical techniques $[15,20,21]$ (interferometry, laser scanning, ...) to measure the top surface topography. The most popular one is a laser scanning technique developed by Flinn et al [15]. The sample surface is scanned by a laser beam and the reflected beam is detected on a position sensitive photodiode located in the focal plane of a lens. This is a very fast technique (the curvature is measured within $5 \mathrm{~ms}$ ) which makes it perfectly compatible with in-situ measurements [22,23]. It is, however, by principle very sensitive to the initial substrate topography: the substrate radius of curvature has to be measured before deposition. A schematic view of the set-up built in MATOP [24] is shown in figure 1.

Concerning multilayered materials the measurement of substrate bending yields the average stress $\langle\sigma\rangle$. Indeed what is measured is the total force per unit length applied by the coating on the substrate:

$$
<\sigma>\sum_{i} t_{i}=\sum_{i} \sigma_{i} t_{i}
$$

where $\sigma_{i}$ and $t_{i}$ are respectively the stress and the thickness of the ith layer.

Moreover, the high density of interfaces present in superlattices make the contribution of interface stresses no more negligible. This will add an additionnal term in expression (2) that will be discussed in section 2.3 .

\section{$2.2 \mathrm{X}$ ray diffraction}

X-ray diffraction and the elastic response of materials provide a powerful method for determining stresses. Stresses alter the spacing of crystallographic planes in crystals by amounts easily measured by diffraction. Let us consider an isotropic body submitted to a biaxial isotropic stress in the $x_{1}-x_{2}$ plane (see figure 2 for the reference frame): $\sigma_{11}=\sigma_{22}=\sigma$. The elastic response of the material is fully determined by two elastic coefficients, the Young modulus $\mathrm{E}$ and the Poisson ratio $v$. It is easy to show that the strain 


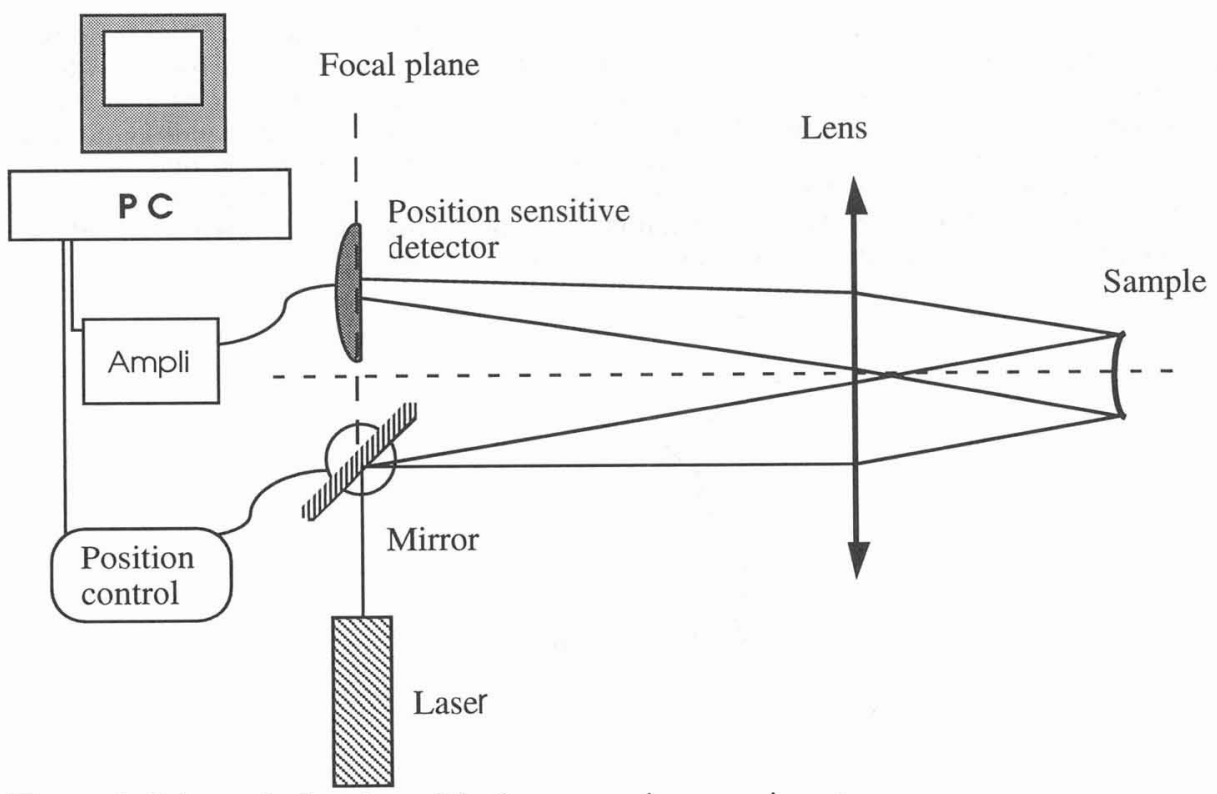

Figure 1: Schematic drawing of the laser scanning experiment

measured in a direction making an angle $\psi$ (figure 2 ) with the normal to the $x_{1}-x_{2}$ plane is given by:

$$
\varepsilon_{\phi \psi}=\frac{1+v}{\mathrm{E}} \sigma \sin ^{2} \psi-2 \frac{v}{\mathrm{E}} \sigma
$$

This is the basis of the $\sin ^{2} \psi$ and related methods. The relation (3) is very general as it applies even to an amorphous material and is a direct consequence of the Poisson elastic effect. The $\sin ^{2} \psi$ method is generally associated with $\mathrm{x}$-rays because crystals lattice spacings, and thus strains, are easily measured by $\mathrm{x}$-ray diffraction. Equation (3) is then used in the new form:

$$
\frac{d_{\psi}-d_{0}}{d_{0}}=\frac{1+v}{\mathrm{E}} \sigma \sin ^{2} \psi-2 \frac{v}{\mathrm{E}} \sigma
$$

By plotting $d_{\psi}$ vs $\sin ^{2} \psi$, one should get a straight line. The slope is directly related to the biaxial stress $\sigma$ and the unstrained lattice spacing $\mathrm{d}_{0}$ corresponds to $\sin ^{2} \psi_{0}=2 v /(1+v)$.

It is not the purpose of this review to go too much into the details of the stress measurements by $\mathrm{x}$-ray diffraction, which is a very specialised and technical subject. Very good books [25] or review papers [26] exist and the interested reader should dig into these references. The very simple approach presented above has been used to outline the general principles underlying the method. The problem is generally more complicate because: 1) A more general state of stress $\sigma_{i j}$ generally occurs. One gets now a system of equations which are solved using as many $d_{h k !}$ measurements as possible. It should be noted that it is not possible to derive simultaneously $\sigma_{33}$ and $a_{0}$, the unstressed lattice parameter, independently. Usually the reasonable assumption: $\sigma_{33}=0$, that the free surface is not subjected to a normal force, is made. 2) The films or multilayers to be studied are generally highly textured or even single crystals. In the case of single crystals, the problem is quite simple. One has to choose the right combination of elastic constants in accordance with the crystal orientation. This case has been excellently reviewed by Clemens and Bain [27]. For example in the case of a cubic material with [111] texture, quite common for fcc metals, one gets:

$$
\varepsilon_{\phi \psi}=\sigma\left\{\frac{1}{2 C_{44}} \sin ^{2} \psi-\frac{C_{11}+2 C_{12}-2 C_{44}}{3 C_{44}\left(C_{11}+2 C_{12}\right)}\right\}
$$


where the $\mathrm{C}_{\mathrm{ij}}$ are the elastic constants in the crystallographic reference frame. In the case of a textured polycrystal the problem is more complex. What is the appropriate elastic constants tensor ? How to extract the average stress tensor from measurements which, by essence, select a family of crystallites in a given orientation? This problem is solved through the use of $\mathrm{x}$-ray elastic constants which are determined from the orientation distribution function of the sample. One has to use an averaging scheme for the elastic constants. The Reuss method assumes that all the crystallites are subjected to the same stress whereas the Voigt method assumes an isostrain state. A more realistic approach is the Kröner-Eshelby self consistent method [28-30] which allows for the continuity of stresses and displacements.

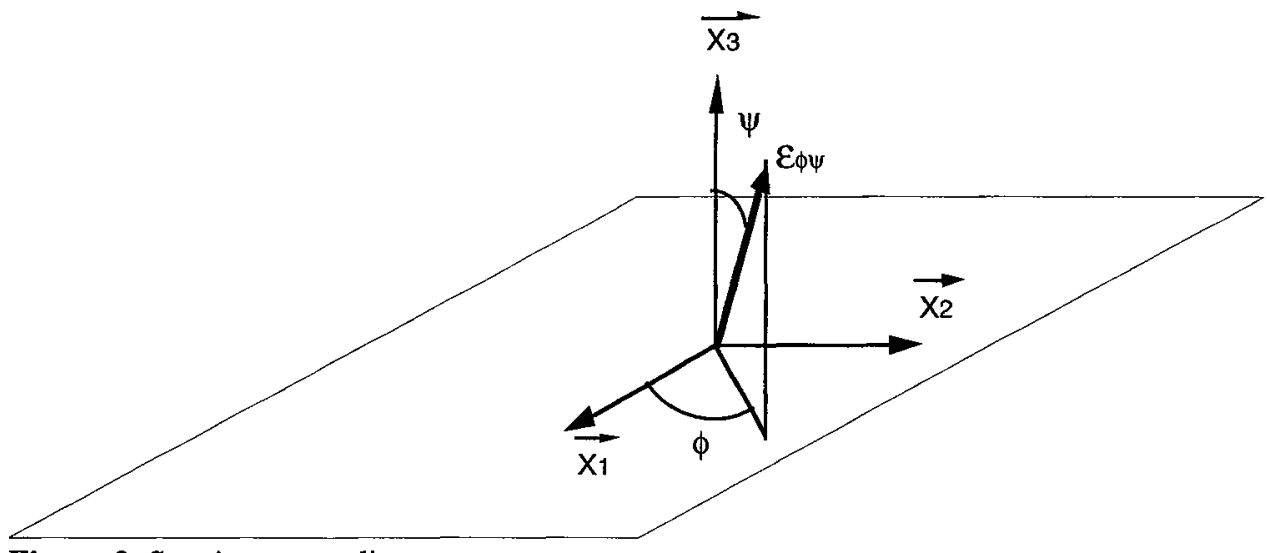

Figure 2: Specimen coordinate system

As was stated above, the $\sin ^{2} \psi$ method as such is valid as long as linear elasticity applies. An important concern, however, in multilayers, is the applicability of x-ray diffraction to the measurement of lattice spacings. A symmetric $\theta / 2 \theta$ scan on a (111) $\mathrm{Au} / \mathrm{Ni}$ multilayer is given in figure 3 . One can clearly see a serie of superlattice peaks, equidistant in the reciprocal lattice, whose intensity is modulated. Although the details of the spectrum call for an understanding of the different kind of disorder present in the stacking [31-34], the general features can easily be understood through the concept of an ideal superlattice consisting of $\mathrm{N}$ bilayers with a period $\Lambda$. Each bilayer is made of $n_{A u}$ planes of Au with spacing $d_{A u}$ and $n_{N i}$ planes of Ni with spacing $d_{N i}$. One has thus: $\Lambda=\left(n_{A u}-1\right) d_{A u}+\left(n_{N i}-1\right) d_{N i}+2 d_{i n t}$ where $d_{i n t}$ is the interfacial distance. Assuming, for simplification, $\mathrm{d}_{\mathrm{int}}=\frac{\mathrm{d}_{\mathrm{Au}}+\mathrm{d}_{\mathrm{Ni}}}{2}$ one gets $\Lambda=\mathrm{n}_{\mathrm{Au}} \mathrm{d}_{\mathrm{Au}}+\mathrm{n}_{\mathrm{Ni}} \mathrm{d}_{\mathrm{Ni}}$. In the kinematical approximation, the amplitude scattered by such a structure is:

$$
F(q)=\sum_{n} f_{n}(q) \exp \left(\text { iqnd }_{n}\right)
$$




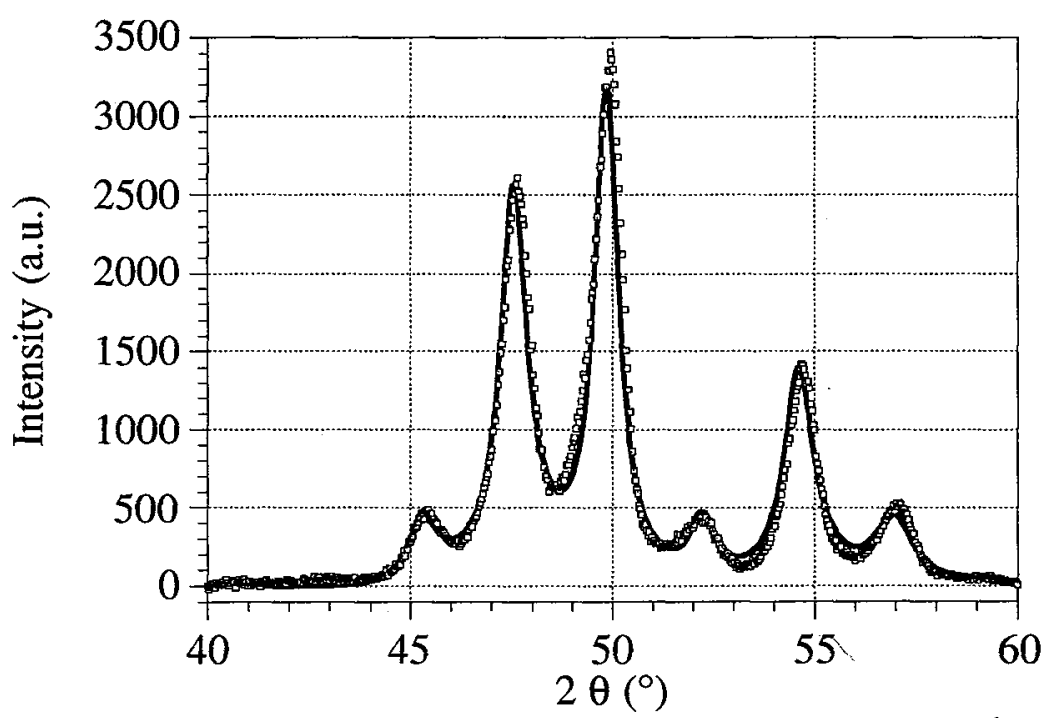

Figure 3: Symmetric $\theta / 2 \theta$ scan $(F e K \alpha)$ from a (111) Au/Ni multilayer $(\Lambda=52 \AA)$. Experimental data (squares) and least squares fit (continuous line) using the algorithm of Fullerton et al [32] are superimposed.

where $q$ is the scattering vector $(q=(4 \pi \sin \theta) / \lambda$ where $\theta$ is the Bragg angle and $\lambda$ is the wavelength of the incident radiation) and $f_{n}$ the scattering factor for plane $n$. This simplifies to $F(q)=S(q) B(q)$ and the scattered intensity is $\mathrm{I}(\mathrm{q})=|\mathrm{S}(\mathrm{q})|^{2}|\mathrm{~B}(\mathrm{q})|^{2}$. IS $\left.(\mathrm{q})\right|^{2}=\frac{\sin ^{2}\left(\frac{N q \Lambda}{2}\right)}{\sin ^{2}\left(\frac{q \Lambda}{2}\right)}$ is a Laue function corresponding to the superlattice. $|\mathrm{B}(\mathrm{q})|^{2}$ is the scattering term which comes from the bilayer and which gives rise to the smooth intensity envelope which appears in the experimental spectrum. An example is given in figure 4 where the different terms are explicited.

In summary the symmetric spectrum consists of a series of peaks separated by $\Delta q=\frac{2 \pi}{\Lambda}$ and whose intensity is proportional to $|\mathrm{B}(\mathrm{q})|^{2}$. This means that the information on $\mathrm{d}_{\mathrm{Au}}$ and $\mathrm{d}_{\mathrm{Ni}}$ lies in the intensity of the superlattice peaks. The position of the peaks is related to the superperiod $\Lambda$ but not to the lattice spacings in the bilayer. One is confronted with a situation very similar to the one of crystallographers looking for interatomic spacings within the crystal basis. Indeed one has to perform intensity refinement and a number of kinematic modeling schemes do exist in the literature [32-34]. In a perfect superlattice, the presence of satellite peaks occurs around every reciprocal lattice node. One should then expect a shift of the Bragg peak positions deduced from diffraction on asymmetrical lines. This would mean that strain measurements on multilayers by $x$-ray diffraction are not possible unless one performs a full modeling of $q_{1}$ scans. Should one conclude that « classical » $\mathrm{x}$-ray diffraction strain measurements on metallic multilayers are not valid? A detailed comparison of stress determination by two different methods [35] has been made on $\mathrm{Au} / \mathrm{Ni}$ multilayers. The first method is a standard strain analysis from the Bragg shift of asymmetrical lines $\left(\sin ^{2} \psi\right.$ method), while the second one uses the perpendicular lattice parameter extracted from the kinematic modeling of a symmetric scan and parallel parameters directly measured from grazing incidence diffraction measurements. The measurement of lattice spacings from planes perpendicular to the multilayer stacking is free from interference fringes. The comparison between these two different methods revealed a good agreement. This means that asymmetric lines are unsensitive to the superlattice modulation whereas symmetric ones are. This results from the large amount of disorder present in these multilayers compared to 
the almost perfect superlattices which are made from semiconductors. The distribution in the number of lattice planes and the small coherence lengths [35] tend to smear out the satellite peaks. On the other hand, the presence of a strong perpendicular preferred orientation ensures a good coherence in the perpendicular direction.

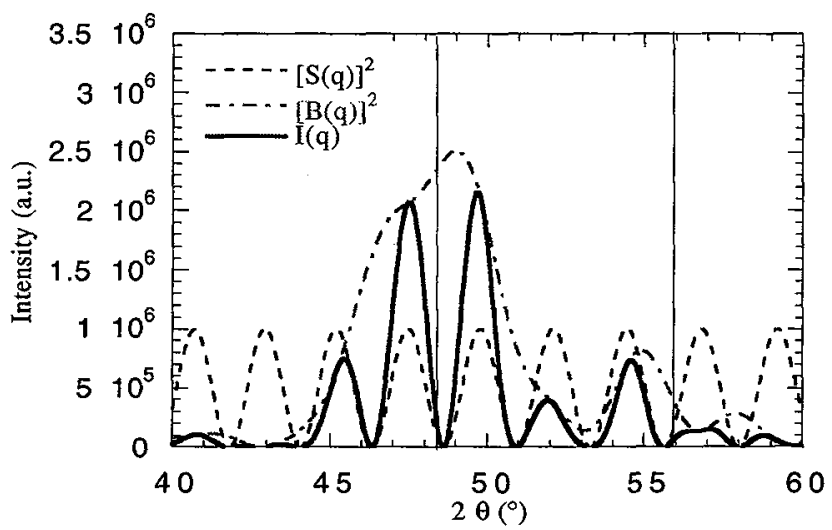

Figure 4: Intensity $I(q)$ diffracted from an ideal $\mathrm{Au} / \mathrm{Ni}$ superlattice. $\mathrm{B}(\mathrm{q})$ is the interference term corresponding to one bilayer and $S(q)$ is the interference function from the superlattice. No disorder was introduced. The peak width $(S(q)$ and $I(q))$ is a size effect: only two bilayers are diffracting. Solid vertical lines indicate the positions expected in the absence of any interferences.

There exist, however, examples of metallic superlattices which exhibit modulated structures away from $\psi=$ $0^{\circ}$. This is the case of $\mathrm{Mo} / \mathrm{V}$ multilayers [36] which, for small enough superperiods, are perfectly epitaxial. One ends up with a beautiful modulated reciprocal superlattice which calls for a detailed refinement.

In conclusion, one should be aware of possible interference effects in metallic superlattices. Reciprocal space mapping is the right way to check for the occurrence of such effects which may strongly influence the stress analysis. The high degree of disorder present in metallic multilayers makes, however, the use of standard stress analysis techniques still valid.

\subsection{Comparison between the two methods}

As pointed out by Nix [9], surface and interface stresses do not result in a film stress but cause substrate bending. The surface stress is the work required to deform elastically a unit area of surface by a unit strain [39]. Whereas in liquids surface stress and surface free energy are numerically equal, they are not in solids. Generally unless one does a careful experimental observation [40], surface stress is a rather negligible quantity for a thin film-substrate composite. In multilayers the great number of interfaces may make the contribution of interfacial stresses no more negligible. The comparison between the substrate interaction stress (deduced from curvature measurements) and the average stress (derived from x-ray diffraction measurements in the two constituents) should yield the interface stress [37,38]. Ruud [37] has measured the interfacial stress in $\mathrm{Ag} / \mathrm{Ni}$ multilayers by using the following expression:

$$
\sigma_{S C} t_{f}=\sigma_{A} t_{A}+\sigma_{B} t_{B}+2 f t_{f} / \Lambda
$$

Where $\sigma_{\mathrm{A}}$ is the stress, as measured by x-ray diffraction, in element $\mathrm{A} ; \mathrm{t}_{\mathrm{A}}$ is the total thickness of element $\mathrm{A}$ and $\mathrm{f}$ is the interfacial stress and $\sigma_{\mathrm{SC}}$ is the substrate interaction stress deduced from curvature measurements. f can thus be extracted via a plot of $\sigma_{\mathrm{SC}}-\left\langle\sigma_{\mathrm{RX}}\right.$ vs $\Lambda$. It relies, however, on a rather drastic assumption: the interfacial structure is assumed to be the same when the superperiod is decreased. As will be discussed below this is not generally the case. Bain et al [41] did not find any evidence for an interfacial stress contribution in $\mathrm{Mo} / \mathrm{Ni}$ superlattices. There is clearly a need for detailed measurements of the stress 
state in metallic multilayers in order to get a better knowledge of the interface stress contribution. From the order of magnitude of interfacial stresses quoted by Cammarata [38], i.e. $1 \mathrm{~J} / \mathrm{m}^{2}$, one gets a $2 \mathrm{f} / \Lambda$ term of about 1 GPa for a $20 \AA$ period, far from being negligible.

\section{MICROSTRUCTURE}

In small period metallic superlattices the high density of interfaces may dominate the microstructure. When the period is reduced, any lattice plane is a few interatomic distances away from an interface. One ends up with a material which is generally very different from what one would expect from a naive abruptinterface picture. The knowledge of the interfacial structure (roughness, lattice strain gradient, interdiffusion, ...) is a fundamental problem. Indeed most of the properties of the superlattices do depend directly on what is going on at interfaces. It calls for the comparison of different kind of measurements ( $\mathrm{x}$ ray scattering, transmission electron microscopy, ...) in order to get information on the $1 \AA$ scale.

It is not possible to discuss here all the different kinds of chemical and structural disorder which may occur in metallic superlattices. There is one phenomenon which seems independent of the growth method and may be intrinsic to multilayers: the unstrained lattice parameter of at least one constituent may differ markedly from the bulk one [42-47]. One may wonder whether reports of a « violation of Poisson effect » $[48,49]$ belong to this kind of effect. Stress analysis through the measurement of asymmetrical lattice parameters is a good way to extract the unstrained lattice spacing. Using this methodology, a number of authors have reported a large shift of the lattice parameter of $\mathrm{Ni}$ in $\mathrm{Au} / \mathrm{Ni}$ [43-46] and in $\mathrm{Ag} / \mathrm{Ni}$ [48], $\mathrm{Pt}$ in $\mathrm{Co} / \mathrm{Pt}$ [47], $\mathrm{Fe}$ in $\mathrm{Fe} / \mathrm{Pt}$ [42] multilayers. The in-plane lattice parameters of $\mathrm{Au}$ and $\mathrm{Ni}$ in (111) $\mathrm{Au} / \mathrm{Ni}$ mulilayers are presented in figure 5 as a function of the superperiod. The intended Au:Ni ratio was $1: 1$. The steeper slope for $\mathrm{Ni}$ may seem surprising, considering the larger biaxial modulus of $\mathrm{Ni}$. It is a consequence of a systematic inflation of the Ni unit cell when the period decreases. For a $52 \AA$ period the unstrained Ni parameter is $1.5 \%$ larger, compared to the bulk. The in-plane tensile strain is $0.38 \%$. This shows clearly that the major effect is the dilatation. What is the origin of this lattice expansion? This is still under debate. One possibility is a mixing between the two constituents. Other authors tend to favor other interpretations in order to explain the lattice parameter shift: electronic effects in these periodic systems have been put forward [52-54] as a possible explanation.

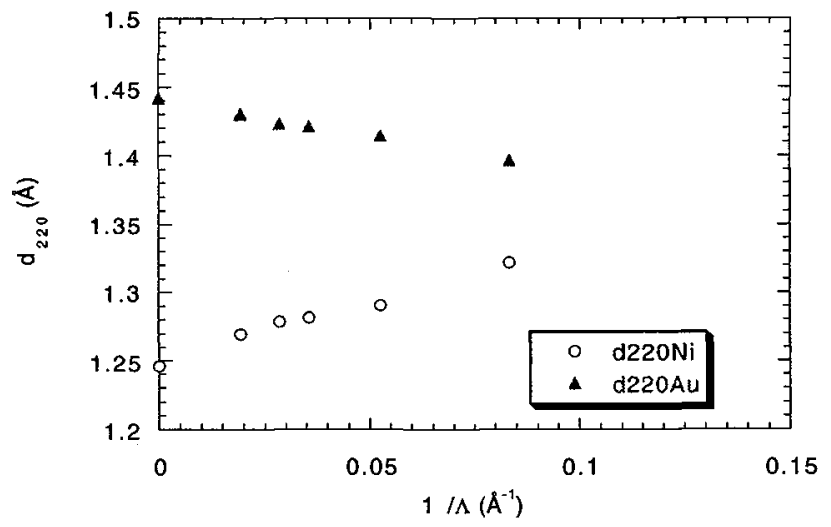

Figure 5: In-plane lattice spacings in (111) Au-Ni multilayers. The values corresponding to an infinite period are the bulk spacings from the literature.

The Au/Ni system, with a limited mutual solubility, has been particularly studied [43-46]. All the authors agree on a systematic shift of the $\mathrm{Ni}$ strain free lattice parameter when the superperiod decreases, whatever the growth technique employed. This large unstrained lattice parameter of $\mathrm{Ni}$ can be consistently interpreted as being a consequence of Au intermixing. Lattice strain analysis from HREM images and EELS analyses [46] tend to support this hypothesis. The Au concentrations needed to support such an interpretation are, however, much larger than the known solubility limit. One may invoke a modification of the phase diagram by the high stress levels present in these superlattices [7]. These multilayers, deposited at - or close to room temperature are probably very far from equilibrium. It is possible therefore that $\mathrm{Au}$ segregation during growth is the reason for the observed Au:Ni intermixing. Molecular Dynamics calculations [51] support this 
hypothesis. A lot of work remains to be done in order to evaluate more quantitatively the effect of segregation during the growth of metallic superlattices, in particular the interplay between growth temperature and growth rate.

\section{RESIDUAL STRESSES}

Very large residual strains, sometimes in excess of 1\%, are reported [41-45] in metallic multilayers. This corresponds to stresses in excess of $1 \mathrm{GPa}$, if bulk elastic moduli are used. For the very large strains encountered this approach may be questioned. Fermi surface and Brillouin zone interaction [55] or large strains [56] have been put forward as possible causes of substantial changes of the elastic constants in metallic superlattices. On the experimental side, as already written in the introduction, the supermodulus effect is no more considered real. Direct measurements of the elastic constants [57-59] show rather modest changes although a softening of $\mathrm{C}_{44}$ in $\mathrm{Ag} / \mathrm{Ni}$ superlattices, up to $40 \%$, is observed when the period is decreased. The very large strains encountered should affect the elastic constants: the asymmetry of interatomic potentials should lead to a stiffening under compression and a softening under tension [56]. When available, one should use second order constants [37].

What is the mechanism responsible for the very high yield stresses encountered in multilayers? We have determined residual stresses as high as $3.9 \mathrm{GPa}$ in Au layers $9 \AA$ thick within Au/Ni multilayers [65]. This is indeed much larger than the reported yield stress for bulk Au: $200 \mathrm{MPa}$. A stress as high as $6.4 \mathrm{GPa}$ has been reported in $42 \AA$ thick $W$ layers within W/Cu multilayers [66]. The small grain size is not the only responsible mechanism. In single films on substrates, the yield strength has been shown to depend on the film thickness. Few detailed studies exist [60-63] where a systematic evaluation of the biaxial yield strength in thin $\mathrm{Pb}, \mathrm{Al}$ or $\mathrm{Cu}$ layers with thicknesses in the $0.1-2 \mu \mathrm{m}$ range has been done. All these studies indicate a clear dependence on thickness in addition to the more usual grain size strengthening mechanism. Several models $[10,64]$ have been proposed which explain high thin film flow stresses and the inverse thickness effect in terms of the stress required for dislocation glide in the constraining presence of the film/substrate interface. In order for flow by dislocation glide to occur, the work done by the stress in the film must also be sufficient to deposit interfacial dislocations. According to Nix [10], this leads to a biaxial yield strength of the form:

$$
\sigma_{\text {yield }}=\frac{\sin \Phi}{\cos \Phi \cos \lambda} \frac{\mathrm{b}}{2 \pi(1-v) \mathrm{t}_{\mathrm{f}}} \frac{\mu_{f} \mu_{s}}{\mu_{f}+\mu_{s}} \ln \left(\frac{\beta_{s} t_{f}}{b}\right)
$$

where $\Phi$ is the angle between the substrate normal and the slip plane, $\lambda$ is the angle between the Burger vector and the substrate normal, $b$ is the Burger vector, $t_{f}$ is the film thickness, $\mu_{f}, \mu_{s}$ are the the elastic shear moduli of the film, and substrate and $\beta_{s}$ is a constant. Doerner [63] has added a grain size strengthening term corresponding to the Hall-Petch relation:

$$
\sigma_{\text {yield, total }}=\sigma_{\text {yield }}+\mathrm{kd} \mathrm{d}^{-\frac{1}{2}}
$$

where $d$ is the grain size. It should be noted, however, that there is some evidence for a $1 / d$ dependance in metallic films rather than the $1 / \mathrm{d}^{1 / 2}$ dependance described by the Hall-Petch relationship. The approach by Thompson [64], takes specifically into account the grain size and leads to the following relationship:

$$
\sigma_{\text {yield, total }}=\frac{\sin \Phi}{\cos \Phi \cos \lambda} \frac{\mathrm{b} \mu_{f}}{4 \pi(1-v)} \ln \left(\frac{\mathrm{d}}{b}\right)\left(\frac{2}{\mathrm{~d} \sin \Phi}+\frac{1}{\mathrm{t}_{\mathrm{f}}}\right)
$$

Theses models provide at least a qualititative explanation for the very impressing stress levels found in metallic multilayers. Detailed studies where the superperiod and the thickness ratio would be varied are welcome. Studies performed on ultra thin films (less than $100 \AA$ ) should be helpful to learn what is really specific to superlattices. Theoretical studies concerning the behaviour of dislocations in ultra thin films would be welcome. 


\section{Conclusion}

In conclusion, the study of residual stresses in metallic multilayers raises a number of interesting problems. Huge strains are encountered and elastic constants calculations are clearly needed. Several thickness dependent phenomena are encountered: residual stresses depend strongly on the thickness of individual films. Strain free lattice parameters, probably linked to segregation during growth, do depend also on the relative thicknesses of the constituents. A great deal of work is still needed to study these phenomena. It is of importance since one is very often looking for period dependent effects in superlattices, as for example interfacial anisotropy (Néel term) in magnetic systems. As was rightly pointed out by [64], one may misinterpret period dependent anisotropy.

Finally, it is a truism to say that multilayers are systems where interfaces are playing a major role. The development of experimental tools for the analysis of the interfacial structure is therefore of paramount importance.

\section{ACKNOWLEDGMENTS}

It is a pleasure to acknowledge B. Pichaud for critical reading of the manuscript.

\section{REFERENCES}

[1] Physics, Fabrication and Applications of Multilayered Structures, edited by P. Dhez and C. Weisbuch (Plenum Press, New York 1988).

[2] Metallic Multilayers, edited by A. Chamberod and J. Hillairet, Material Science Forum, 59-60 (1990).

[3] R. Naik, A. Poli, D. McKague, A. Lukaszew, L.E. Wenger, Phys. Rev. B 51 (1995) 3549.

[4] W.M.C. Yang, T. Tsalakakos, and J.E. Hilliard, J.Appl. Phys. 48 (1977). 876.

[5] M.K. Small, B.J. Daniels, B.M. Clemens and W.D. Nix, J.Mater. Res. 9 (1994) 25.

[6] S.P. Baker and W.D. Nix, J.Mater. Res. 9 (1994) 3131.

[7] J.W. Cahn and F. Larché, Acta Metal. 32 (1984) 1915.

[8] G.A. Prinz, Phys. Rev. Lett. 54 (1985) 1051.

[9] M.F. Doerner, W.D. Nix, Critical Reviews in Solid State and Materials Sciences 14 (1988) 225.

[10] W.D. Nix, Metall. Trans. A 20 (1989) 2217.

[11] H. Windischmann, Critical Rviews in Solid State and Materials Sciences 17 (1992) 547.

[12] G. Stoney, Proc. Roy. Soc. London, Ser.A 82 (1909) 172.

[13] P.H. Townsend, D.M. Barnett, T.A. Brunner, J. Appl. Phys. 62 (1987) 4438.

[14] S. N. G. Chu, A.T. Mackrander, K.E. Strege, and W.D. Johnston Jr, J. Appl. Phys. 57 (1985) 249.

[15] P. Flinn, D. Gardner, W. Nix, IEEE Trans. Elec. Devices ED-34 (1987) 689.

[16] P.M. Marcus, submitted to Phys. Rev. B.

[17] W. Brantley, J. Appl. Phys. 44 (1973) 534.

[18] A. Segmüller, J. Angilelo, S.J. LaPlaca, J. Appl. Phys. 51 (1980) 6224.

[19] N. Burle, B. Pichaud, N. Guelton, R.G. Saint-Jacques, Thin Solid Films 260 (1995) 65

[20] M. Jarosz, L. Kocsanyi, J. Giber, J. Phys. E: Sci. Instrum. 15 (1982) 746.

[21] S.T. Chen, C.H. Yang, F. Faupel, P.S. Ho, J. Appl. Phys. 6 (1988) 6690.

[22] R. Abermann, Mat. Res. Soc. Symp. Proc. 239 (1992) 25.

[23] T.F. Ueda, G.F. Simenson, W.D. Nix, and B.M. Clemens, Mat. Res. Soc. Symp. Proc. 382 (1995) 279.

[24] S. Labat, Diplome d'Etudes Approfondies, Université Aix-Marseille III, 1994.

[25] I. Noyan, J. Cohen, Residual stresses, Spinger Verlag editors, Stuttgart 1987.

[26] I.C Noyan, T.C. Huang, and B.R. York, Critical Reviews in Solid State and Materials Sciences 20 (1995) 125.

[27] B.M. Clemens and J.A. Bain, Mat.Res. Bull. 17 (1992) 46.

[28] M. Francois, PhD thesis, ENSAM Paris 1991.

[29] E. Kröner, Z. für Physik, 151 (1958) 504.

[30] J. Eshelby, Proc. Roy. Soc. A241 (1957) 376.

[31] A. Segmüller and A.E. Blakeslee, J. Appl. Cryst. 6 (1973) 19.

[32] E. Fullerton, I. Schuller, H. Vanderstraeten, Y. Bruynseraede, Phys. Rev. B 45 (1992) 9292

[33] F. Lamelas, H. David He, R. Clarke, Phys. Rev. B 43 (1991) 12296.

[34] G. Gladyszewski, Thin Solid Films 204 (1991) 473.

[35] S. Labat, O. Thomas, L. Barrallier, B. Gilles, A. Marty, G. Patrat, X'Top 96 Palermo, to appear in 
Nuovo Cimento.

[36] J. Birch, J.E. Sundgren, P.F. Fewster, J. Appl. Phys. 78 (1995) 6562.

[37] J.A. Ruud, A. Witrouw, F. Spaepen, J. Appl. Phys. 74 (1993) 2517.

[38] R.C. Cammarata, Prog. Surf. Sci. 46 (1994) 1.

[39] W.W. Mullins, in Metal Surfaces (American Society for Metals, Metals Park, OH, 1962), pp17-66.

[40] P. Müller, R. Kern, Surf. Sci. 301 (1994) 386.

[41] J.A. Bain, L.J. Chung, S. Brennan, and B.M. Clemens, Phys. Rev. B 44 (1991) 1184.

[42] B.J. Daniels, W.D. Nix and B.M. Clemens, Mat.Res. Soc. Symp. Proc. 356 (1995) 373.

[43] N. Nakayama, L. Wu, H. Dohonomae, T. Shinjo, J. Kim and C.M. Falco, JMMM 126 (1993) 71

[44] S. Baker, J. Bain, B. Clemens, W. Nix, Mat. Res. Soc. Proc. 343 (1994) 555.

[45] S. Labat, B. Pichaud, O. Thomas, C. Alfonso, A. Charaï L. Barrallier, B. Gilles, A. Marty, EMRS 1995, Strasbourg. To appear in Thin Solid Films.

[46] P. Bayle, T. Deutsch, B. Gilles, F. Lancon, A. Marty, J. Thibault, C. Colliex, M. Tence, Mat. Res. Soc. Symp. Proc. 319 (1994).

[47] J.A. Bain, B.M. Clemens, H. Notarys, E;E. Marino, S. Brennan, J. Appl. Phys. 74 (1993) 996.

[48] K.F. Badawi, N. Durand, P. Goudeau, V. Pelosin, Appl. Phys. Lett. 65 (1994) 3075.

[49] A.F. Jankowski, J. Appl. Phys. 71 (1992) 1782.

[50] A. Fartash, M. Grimsditch, E.F. Fullerton, I.K. Schuller, Phys. Rev. B 49 (1994) 12813.

[51] J. Eymery, F. Lançon, L. Billard, J. Physique I 3 (1993).

[52] P. Jonnard, F. Vergand, C. Bonnelle, K.F. Badawi, J. Appl. Phys. 77 (1995) 6044.

[53] C.S. Baxter, W.M. Stobbs, Nature 322 (1986) 814.

[54] S.J. Lloyd, R.E. Somekh, W.M. Stobbs, Mat. Res. Soc. Symp. Proc. 382 (1995) 155.

[55] W.E. Pickett, J. Phys. F: Met. Phys. 12 (1982) 2195.

[56] A.F. Jankowski and T. Tsalakos, J. Phys. F: Met. Phys. 15 (1985) 1279.

[57] G. Carlotti, D. Fioretto, G. Socino, B. Rodmacq, V. Pelosin, J. Appl. Phys. 71 (1992) 4897.

[58] G. Carlotti, G. Socino, A. Hu, H. Xia, S. Jiang, J. Appl. Phys. 75 (1994) 3081.

[59] G. Carlotti, G. Socino, A. Hu, H. Xia, S. Jiang, Mat. Res. Soc. Symp. Proc. 343 (1994) 567.

[60] M. Murakami, Acta Met. 26 (1978) 175.

[61] R.P. Vinci, E.M. Zielinski, J.C. Bravman, Mat. Res. Soc. Symp. Proc. 356 (1995) 459.

[62] R. Venkatraman, J.C. Bravman, J. Mater. Res. 7 (1992) 2040.

[63] M.F. Doerner, D.S. Gardner, and W.D. Nix, J. Mater. Res. 1 (1986) 845.

[64] C. Thompson, J. Mater. Res. 8 (1993) 237.

[65] B.M. Clemens, R.L. White, W.D. Nix, J.A. Bain, Mat. Res. Soc. Symp. Proc. 231 (1992) 459.

[66] S. Labat, P. Gergaud, O. Thomas, unpublished results.

[67] P. Goudeau, K.F. Badawi, A. Naudon, G. Gladyszewski, Appl. Phys. Lett. 62 (1993) 246. 